\title{
VDAC contributes to mRNA levels in Saccharomyces cerevisiae cells by the intracellular reduction/oxidation state dependent and independent mechanisms
}

\author{
Hanna Galgańska • Monika Antoniewicz • \\ Małgorzata Budzińska • Kukasz Gałgański • \\ Hanna Kmita
}

Received: 30 June 2010 / Accepted: 19 October 2010 /Published online: 12 November 2010

(C) The Author(s) 2010. This article is published with open access at Springerlink.com

\begin{abstract}
Available data suggest that voltage-dependent anion selective channel (VDAC) constitutes an important component of a cellular regulatory mechanism based on the intracellular reduction/oxidation (redox) state. Here, using quantitative RT-PCR, we demonstrated that depletion of VDAC1 (termed here VDAC) in Saccharomyces cerevisiae cells distinctly affected levels of mRNAs encoding nuclear proteins sensitive to changes of the intracellular redox state including the nuclear transcription factors important for adaptation to the redox state and proteins involved in communication between mitochondria and the nucleus. We also revealed that the changes of the studied protein transcript levels generally correlated with changes of the intracellular redox state although VDAC appears also to affect mRNA levels by a mechanism not based on changes of the intracellular redox states. Thus, VDAC seems to be an important element of the intracellular signaling network.
\end{abstract}

Keywords Saccharomyces cerevisiae.

Quantitative RT-PCR · VDAC · Reduction/oxidation state

H. Gałgańska $\cdot$ M. Antoniewicz $\cdot$ M. Budzińska $\cdot H$. Kmita $(\bowtie)$ Laboratory of Bioenergetics, Institute of Molecular Biology and Biotechnology, Faculty of Biology, Adam Mickiewicz University, Umultowska 89,

61-614, Poznań, Poland

e-mail:kmita@amu.edu.pl

Ł. Gałgański

Department of Biotechnology, Institute of Molecular Biology and Biotechnology, Faculty of Biology, Adam Mickiewicz University, Umultowska 89,

61-614, Poznań, Poland

\begin{abstract}
Abbreviations
RT-PCR real-time polymerase chain reaction

VDAC voltage-dependent anion selective channel
\end{abstract}

\section{Introduction}

A growing number of data show that mitochondrial functions are regulated or even governored by voltage-dependent anion selective channel (VDAC) (Lemasters and Holmuhamedov 2006; Mannella and Kinnally 2008; Rostovtseva and Bezrukov 2008). VDAC, also known as mitochondrial porin, was identified in 1976 (Schein et al. 1976) and since that time has been studied extensively. VDAC is the main channel of the outer mitochondrial membrane and it may be present as isoforms encoded by separated genes, displaying different channel-forming activities and probably playing different roles (for reviews see, for example, Benz 1994; Blachly-Dyson and Forte 2001; De Pinto et al. 2003; Colombini 2004; Mannella and Kinnally 2008; ShoshanBarmatz et al. 2008, 2010) Physiologically, VDAC functions as a major channel allowing passage of metabolites between the intermembrane space of mitochondria and the cytosol. The simple transport function is indispensable for proper mitochondrial functioning and, consequently for cell activity, and makes VDAC crucial for a range of cellular processes including ATP rationing, $\mathrm{Ca}^{2+}$ homeostasis and apoptosis execution.

It has been also shown that VDAC mediates the intracellular reduction/oxidation (redox) states (Budzinska et al. 2007; Galganska et al. 2008). Moreover, it is proposed 
that VDAC participates in a reduction/oxidation mechanism important for communication between mitochondria and the nucleus (Galganska et al. 2010). It is well known that the intracellular redox states affect expression, stabilization, localization, accessibility, interactions and activity of cellular proteins and consequently may contribute to communication between mitochondria and the nucleus (e.g. Butow and Avadhani 2004; Liu et al. 2005; Storz 2006; Pesaresi et al. 2007; Woodson and Chory 2008; Galganska et al. 2010). Changes of cellular protein expression levels are usually preceded by changes of their respective mRNA levels. Interestingly, it has been shown that Saccharomyces cerevisiae cells cultured in the presence of an oxidant, which results in changes of the intracellular redox states (e.g. Galganska et al. 2008), display changes of levels of mRNA encoding nuclear transcription factors involved in adaptation to the redox state (Msn2 and Msn4) and Rtg2, a cytoplasmic protein participating in communication between mitochondria and the nucleus (Aragon et al. 2006). Therefore, one could expect a VDAC effect on levels of mRNAs encoding nuclear proteins sensitive to changes of the intracellular redox states involving the nuclear transcription factors as well as proteins involved in communication between mitochondria and the nucleus.

S. cerevisiae is a convenient model to investigate the functional relationship between VDAC, the intracellular redox states and expression levels of cellular proteins. First of all, S. cerevisiae mitochondria express two VDAC isoforms, of which only one has been proved to form a channel (Blachly-Dyson et al. 1997; Lee et al. 1998). The VDAC isoform encoded by the POR1 gene is called VDAC1 (or porin 1) and its properties are highly conserved in other species. Moreover, wild type and VDAC1-depleted mutant of $S$. cerevisiae ( $\triangle$ porl mutant) display differences in expression levels of cellular proteins, also at the level of their encoding mRNAs, that coincide with the cytosol and mitochondrial redox states (Kmita et al. 2004; Budzinska et al. 2007; Galganska et al. 2008). Therefore, we evaluated the effect of VDAC1 (termed here VDAC) disruption in $S$. cerevisiae cells on levels of mRNAs encoding nuclear proteins sensitive to changes of the intracellular redox states including the nuclear transcription factors as well as proteins involved in communication between mitochondria and the nucleus We found that the absence of VDAC resulted in a distinct decrease in relative levels of their encoding mRNAs. We also revealed that the observed changes of mRNA levels correlated with changes of the intracellular redox states, although VDAC appeared also to affect mRNA levels by a mechanism not based on changes of the intracellular redox states. Nevertheless, the involvement of VDAC in the regulatory mechanisms seems to be consistent with data pointing at VDAC as an important element of intracellular signaling (Huizing et al. 1996; Lemasters and Holmuhamedov 2006; Pesaresi et al. 2007; Walther et al. 2009; Shoshan-Barmatz et al. 2008, 2010).

\section{Material and methods}

Yeast strains and culture conditions The following Saccharomyces cerevisiae strains were used: the isogenic wild type M3 (MATa, lys2 his4 trp1 ade2 leu2 ura3) and VDAC1 (porin1)-depleted mutant M22-2 ( $\Delta$ por1) (Blachly-Dyson et al. 1997; Lee et al. 1998). Yeast cells were grown at $28^{\circ} \mathrm{C}$ in YPG medium ( $1 \%$ yeast extract, $2 \%$ peptone, $3 \%$ glycerol) at $\mathrm{pH} 5.5$ to exponential phase $\left(\mathrm{OD}_{546}\right.$ of $\left.0.9-1.1\right)$ or to stationary phase $\left(\mathrm{OD}_{546}\right.$ of 2.0-2.3). For modification of exponential phase $10 \mu \mathrm{M}$ menadione (wild type) or $10 \mathrm{mM}$ ascorbate $(\Delta$ por 1$)$ were added to a given cell culture at 0.1 OD and cells were grown until standard exponential phase (Galganska et al. 2008).

Isolation of mitochondrial and cytosol fractions and determination of their redox states Yeast cells in a given growth phase were converted to spheroplasts, and mitochondria and postmitochondrial supernatant which is largely cytosolic (Sturtz et al. 2001) were isolated according to a previously published procedure (Daum et al. 1982). The redox state of cytosols and mitochondria were determined by calculation of the GSH/total glutathione ratio. The total glutathione (GSH + GSSG) and GSH concentrations (in $\mathrm{nmol} / \mathrm{mg}$ protein) were determined as described previously (Galganska et al. 2008), in the presence of glutathione reductase and glyoxalase I, respectively. The obtained values were tested by $t$ test and they turned out to be significantly different.

Extraction of total RNA Yeast cells in a given growth phase were homogenized in the presence of TRIZOL reagent (Invitrogen). Total RNA was extracted using chloroform and dissolved in RNase-free water. The extracted RNA was quantified using a ND-1000 NanoDrop spectrophotometer. To eliminate genomic DNA contamination, an additional DNase treatment was performed according to standard procedure. The absence of contaminant genomic DNA in RNA preparations was verified using them as a template in PCR assays.

Quantitative RT PCR Primers for RT-PCR (Table 1) were designed using Web primer interface at SGD (Saccharomyces Genome Database, http://www.yeastgenome.org) and used to obtain unique products ranging from 82 to $149 \mathrm{bp}$ and located near the $3^{\prime}$ end of cDNA sequences. Total RNA was reverse-transcribed into cDNA using RevertAid ${ }^{\mathrm{TM}} \mathrm{H}$ Minus First Strand cDNA Synthesis Kit 
Table 1 Primers used for quantitative RT-PCR

\begin{tabular}{|c|c|c|}
\hline Name & Primer sequence & Annealing temperature $\left[{ }^{\circ} \mathrm{C}\right]$ \\
\hline$R T G 1$ & $\begin{array}{l}\text { F: AAGCCCAACAAGGGCCAAATTCT } \\
\text { R: TTGACAATGGTGCCTGTCTGCTT }\end{array}$ & 57 \\
\hline$R T G 2$ & $\begin{array}{l}\text { F: CCAACAGCTGCATTACATGTTGC } \\
\text { R: CAATTCCTATCAGCGCTCTAGCT }\end{array}$ & 57 \\
\hline SKN7 & $\begin{array}{l}\text { F: CAGCGACATCCATTGTCAGAAGTT } \\
\text { R: GTGTAAATCATCCCTCGTGAATGG }\end{array}$ & 57 \\
\hline MSN2 & $\begin{array}{l}\text { F: AGTGTCAACTCAACTGGCAATGGT } \\
\text { R: CTTCTGGACGGTGTCATTGATTTTC }\end{array}$ & 57 \\
\hline MSN4 & $\begin{array}{l}\text { F: AATTGGCGACTTCTGGTGTTGATA } \\
\text { R: GTTGATGATGTTGAGCTGCATGG }\end{array}$ & 57 \\
\hline$Y A P 1$ & $\begin{array}{l}\text { F: CAATCGGTACCAGGAAATGAAAGC } \\
\text { R: CCTTCCTTAGATGGAACGACATCA }\end{array}$ & 57 \\
\hline$Y A P 2$ & $\begin{array}{l}\text { F: AGTGTATCGGGACCAAACCATGTT } \\
\text { R: TGCTTCAGATTGGGTTCGAATTGT }\end{array}$ & 57 \\
\hline SIR2 & $\begin{array}{l}\text { F: ATCACATTCTTTGGCGAAGCACT } \\
\text { R: GAACGTGGGAAGGAACCATGTT }\end{array}$ & 57 \\
\hline$H A P 1$ & $\begin{array}{l}\text { F: ACAAGGCTCAGACTTAGCATCCAA } \\
\text { R: AGGATCGTTGTATCCACCAACAAC }\end{array}$ & 57 \\
\hline$G P X F$ & $\begin{array}{l}\text { F: CAAGAACCTGGCTCTGATGAAGA } \\
\text { R: AACTTGTAAACAGGGTCCTCATTG }\end{array}$ & 57 \\
\hline PUF3 & $\begin{array}{l}\text { F: GTCCTGGAATTATCTGACTCCGT } \\
\text { R: TGGGTAGATAGATGGTAAATGTGG }\end{array}$ & 57 \\
\hline TOM40 & $\begin{array}{l}\text { F: AAATGTCGAGGCAGGTATCGAAAC } \\
\text { R: TTAGCACCAATAGTGGTAGAACCCT }\end{array}$ & 61 \\
\hline$A C T 1$ & $\begin{array}{l}\text { F: CCACCATGTTCCCAGGTATTGC } \\
\text { R: CACCAATCCAGACGGAGTACTTTC }\end{array}$ & 63 \\
\hline$M G E 1$ & $\begin{array}{l}\text { F: CAATGGCACCAAGAACTCCTTTTG } \\
\text { R: CTTTGGATTCTTCACTTTTGGCTTC }\end{array}$ & 63 \\
\hline
\end{tabular}

Forward (F) and reverse (R) primer sequences are shown in $5^{\prime}$ to $3^{\prime}$ orientation. were tested by $t$ test and they turned out to be significantly different.

Other methods Protein concentrations were measured by the method of Bradford and albumin bovine serum, essentially fatty acid free, was used as a standard. To estimate quality of isolated mitochondria respiration of the mitochondria was monitored at $25{ }^{\circ} \mathrm{C}$ with a Rank oxygen electrode in an incubation volume of $0.5 \mathrm{ml}$.

\section{Results and discussion}

Transcript levels of nuclear proteins sensitive to changes of the intracellular redox states including the nuclear transcription factors as well as proteins involved in communication between mitochondria and the nucleus are significantly affected in the absence of VDAC

We have shown that voltage-dependent anion selective channel (VDAC) takes part in the determination of the intracellular redox states, which is important for the redox regulation of expression and activity of cellular proteins such as proteins that participate in protein import into 
mitochondria and antioxidant enzymes (Galganska et al. 2010). To further investigate the involved mechanism, we decided to study the effect of VDAC presence on transcript levels of proteins that take part in mitochondria/nucleus communication and nuclear proteins sensing of the redox state including transcription factors that participate in adaptation to the redox state change. Therefore we applied quantitative RT-PCR to compare levels of their encoding mRNAs in cells of $S$. cerevisiae $\Delta$ porl mutant depleted of VDAC1 (termed here VDAC) and the isogenic wild type. The studied proteins included proteins participating in mitochondria/nucleus communication (Rtg1, Rtg2), nuclear transcription factors participating in adaptation to the redox state change (Msn2, Msn4, Yap1, Yap2, Skn7) and Sir2, a nuclear sensor of the redox state (Cabiscol et al. 2000; Butow and Avadhani 2004; Drakulic et al. 2005; Liu et al. 2005; Lushchak 2010). As control proteins we used Hap4, a nuclear transcription factor controlling genes of aerobic metabolism (Brons et al. 2002), Gpx, a cytoplasmic sensor of the redox state (Liu et al. 2005), Puf3 that controls transport and stability of mRNAs encoding proteins imported into mitochondria (García-Rodríguez et al. 2007) and Tom40, a subunit of the TOM complex being a part of the mitochondrial protein import machinery located in the outer membrane of mitochondria (for reviews see, for example, Dolezal et al. 2006; Bohnert et al. 2007; Mokranjac and Neupert 2009). The expression of Tom40 at the level of both encoding mRNA and protein has been shown to be affected by VDAC depletion (Kmita et al. 2004; Budzinska et al. 2007; Galganska et al. 2008). As reference genes, we applied $A C T 1$ encoding actin, traditionally used in expression studies of $S$. cerevisiae by Northern blot or quantitative RT-PCR (e.g. RodríguezQuiñones et al. 2008) and $M G E 1$ encoding mitochondrial GrpE protein which expression remains unchanged over a wide range of redox conditions (Budzinska et al. 2007; Galganska et al. 2008). These genes belong to different functional categories that minimizes the risk of coregulation. Importantly, the relative mRNA levels calculated with $A C T 1$ and MGE1 were comparable. Therefore, we used both reference genes in further calculations. Moreover, this denotes that $M G E 1$ can be applied as the reference gene in quantitative RT-PCR studies concerning $S$. cerevisiae cells.

We performed RT-PCR experiments for cells in exponential, stationary and modified exponential growth phases (Galganska et al. 2008). The latter denotes an exponential growth phase modified toward stationary growth phase with regard to the cytosol and mitochondria redox states. For the modification, $10 \mu \mathrm{M}$ methadone or $10 \mathrm{mM}$ ascorbate were added to wild type and $\Delta$ porl cells, respectively, at the early exponential growth phase and cells were grown until standard exponential phase (Galganska et al. 2008). As shown in Fig. 1, for all growth phases, levels
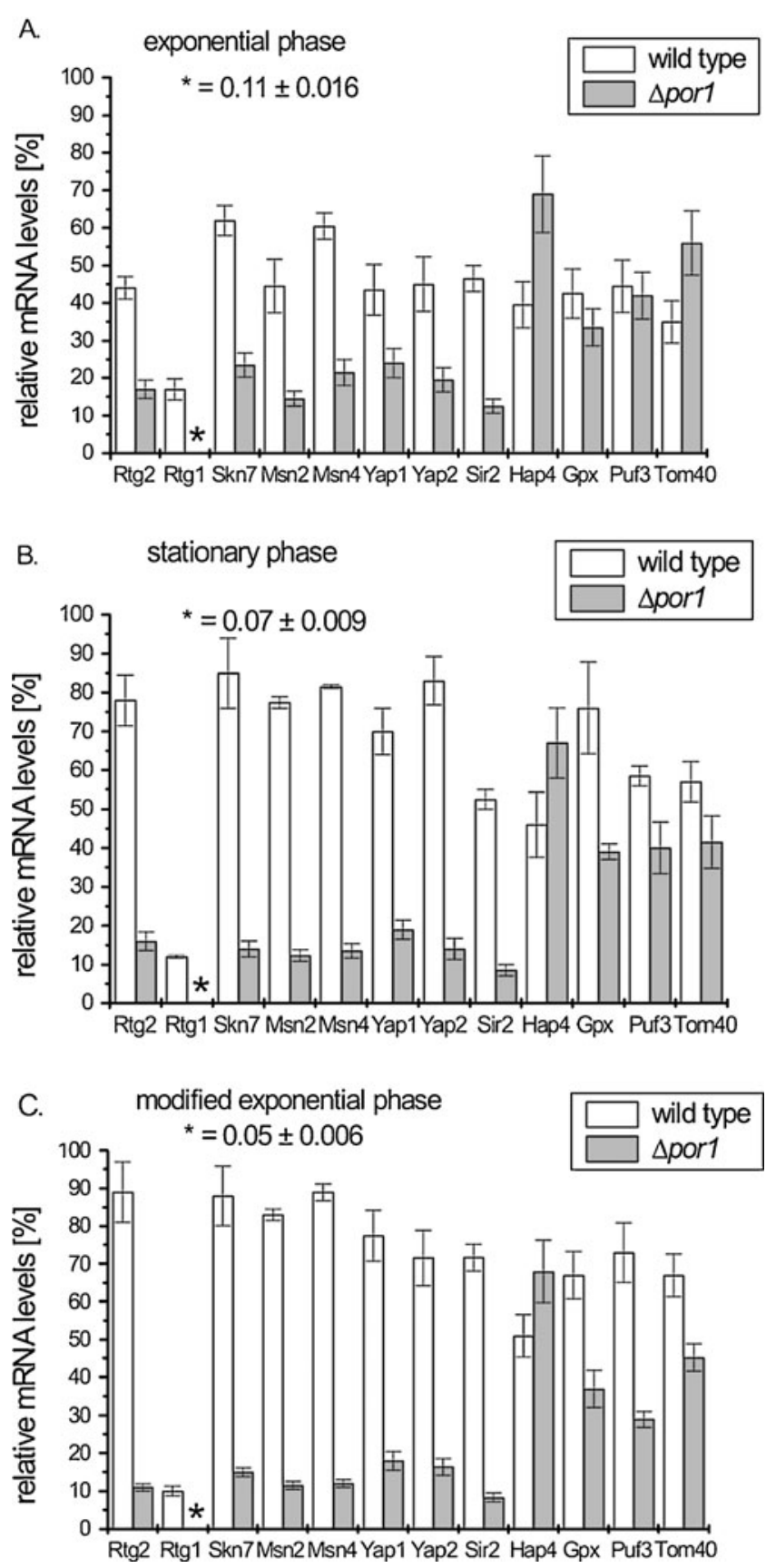

Fig. 1 Relative mRNA levels of selected genes in wild type and $\Delta$ porl S. cerevisiae cells in exponential (a), stationary (b) and modified exponential (c) growth phases. ACT1 and $M G E 1$ were used as reference genes. The relative mRNA levels were determined using mRNA levels calculated by SDS 2.3 software (Applied Biosystems). Data are mean values \pm SEM of $n=9$ RT-PCR reactions (3 experiments with 3 replicates per an experiment). Statistical significance was tested by $t$ test (at $\alpha=0.01$ )

of mRNAs encoding Rtg1, Rtg2, Skn7, Msn2, Msn4, Yap1, Yap2 and Sir2 were significantly down-regulated in the absence of VDAC ( $\Delta$ por 1 mutant) when compared to Hap4, Gpx, Puf3 and Tom40. The decrease in transcript levels was 
particularly visible for Rtg1, a transcription factor participating in communication between mitochondria and the nucleus and located in both nucleus and cytoplasm. Moreover, the down-regulation was obtained for both exponential and stationary growth phase cells (Fig. 1a,b), which indicates that the observed decrease is not transient and connected to a given growth phase but immanently results from the absence of VDAC. On the other hand, the up-regulation of mRNA encoding Hap4 observed for $\Delta$ porl cells in both growth phases indicate that a decrease in transcript levels is not a general feature of a mutant devoid of VDAC. Thus, the absence of VDAC results in clear down-regulation of mRNAs encoding proteins participating in mitochondria/ nucleus communication (Rtg1, Rtg2) and nuclear proteins sensing the redox state (Sir2) and participating in adaptation to the redox state change (Skn7, Msn2, Msn4, Yap1, Yap2). Interestingly, levels of mRNA encoding Gpx regarded as the redox state sensor in cytoplasm is much less sensitive to the absence of VDAC. The same concerns Puf3 and Tom40, although in the case of the latter it has been reported that the protein level depends on the presence of VDAC as well as the cell growth phase and the actual cytosol redox state (Budzinska et al. 2007; Galganska et al. 2008). Importantly, for modified exponential growth phase (Fig. 1c) the obtained relative mRNA levels for both wild type and $\Delta$ porl cells were more similar to those obtained for stationary growth phase (Fig. 1b) than to the data calculated for exponential growth phase (Fig. 1a). Thus, the changes of mRNA levels appear to correlate with the intracellular redox states.

VDAC affects transcript levels via redox state dependent and independent mechanisms

Using the $S$. cerevisiae model system we have shown that VDAC mediates the intracellular redox states in a growth phase dependent way (Budzinska et al. 2007; Galganska et al. 2008, 2010). Thus, for wild type and VDAC-depleted mutant ( $\Delta$ porl mutant) the growth phases differ in the intracellular redox states. As shown in Fig. 2, the calculated values of relative mRNA level ratios between stationary and exponential growth phases (Fig. 2a) as well as modified exponential and exponential growth phases (Fig. 2b) were comparable and differed distinctly for wild type and $\Delta$ por 1 mutant with exception of Rtg1 and Hap4. The value of the ratio smaller than 1 denotes a decrease in relative mRNA levels during stationary or modified exponential growth phase in comparison with exponential growth phase, whereas the value of the ratio higher than 1 denotes an increase in relative mRNA levels during stationary or modified exponential growth phase in comparison with exponential growth phase. Therefore, it can be concluded that during stationary and modified exponential growth phases transcript levels of Rtg1 decreases and transcript
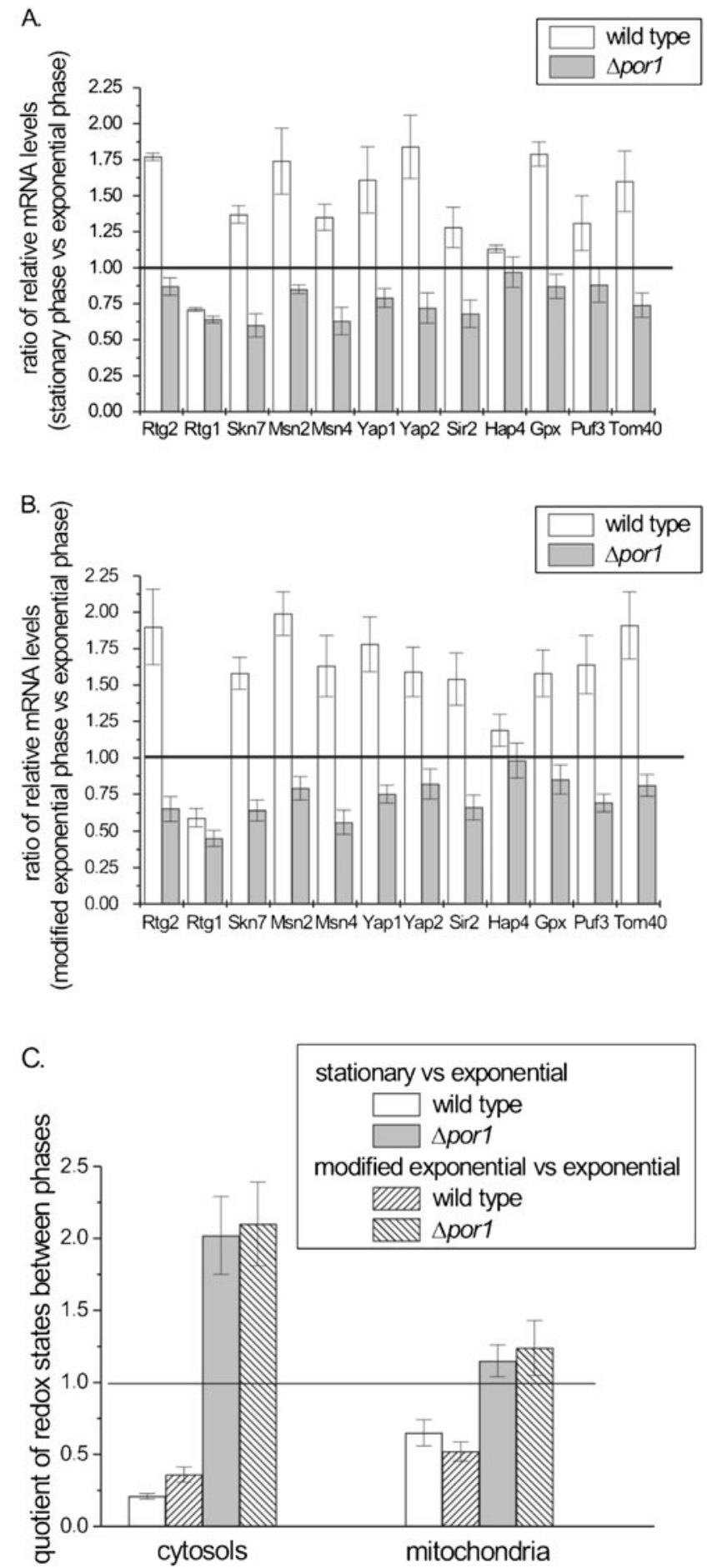

Fig. 2 Changes of the relative levels of mRNA encoding selected proteins versus changes of the intracellular redox states between different growth phases of wild type and $\Delta$ porl S. cerevisiae cells. a Stationary to exponential phase ratio and $\mathbf{b}$ modified exponential to exponential phase ratio concerning relative levels of mRNA encoding the studied proteins for wild type and $\Delta$ porl cells. Data shown in Fig. 1 were used to prepare parts a and b, respectively. $\mathbf{c}$ Stationary to exponential phase and modified exponential to exponential phase quotient of redox states for wild type and $\Delta$ porl cytosols and mitochondria. Data are mean values \pm SEM of three independent experiments performed for each type of cells 
level of Hap4 does not change in both wild type and $\Delta$ por 1 cells. On the other hand, transcript levels of Rtg2, Sir2, Skn7, Msn2, Msn4, Yap1, Yap2, Gpx, Puf3 and Tom40 decrease in $\Delta$ porl cells and increase in wild type cells. The difference in transcript level change is the most pronounced for Sir2, Skn7, Msn2, Msn4, Yap1 and Yap2.

Next we investigated correlation between observed changes of transcript levels and changes of the intracellular redox states. For this purpose, we calculated GSH/total glutathione ratios, i.e. the redox states for wild type and $\Delta$ por1 mitochondria and cytosols isolated from cells in exponential, stationary and modified exponential growth phases. Then, we calculated values of quotients of the redox states between stationary and exponential growth phases as well as modified exponential and exponential growth phases for the studied types of mitochondria and cytosols (Fig. 2c). The value of the quotient smaller than 1 denotes lower redox state (i.e. higher oxidation) during stationary or modified exponential growth phase in comparison with exponential growth phase. By analogy, the value of the quotient a higher than 1 denotes higher redox state (i.e. higher reduction) during stationary or modified exponential growth phase in comparison with exponential growth phase. A decrease in the ratio of relative mRNA levels (Fig. 2a,b) observed for $\Delta$ porl cells corresponds well with the quotient of the redox state calculated for cytosol but not with that calculated for mitochondria (Fig. 2c). For wild type cells, an increase in the ratio of relative mRNA levels (Fig. 2a,b) also correlates well with the quotient of the redox state calculated for cytosol, although the redox state of mitochondria cannot be excluded (Fig. 2c). However, in the case of Rtg1 and Hap4 no effect of the intracellular redox state was observed. Thus, in the case of these proteins, VDAC appears to affect the relative levels of their transcripts by a mechanism that does not include changes of the intracellular redox states. On the other hand, levels of mRNAs encoding Rtg2, Sir2, Skn7, Msn2, Msn4, Yap1, Yap2, Gpx, Puf3 and Tom40 increase when the intracellular redox state, mainly the cytosol redox state, shifts towards oxidation. It is known that in response to oxidative stress, cells activate expression of a number of genes. In many cases, these induced genes encode transcription factors whose expression, structure, subcellular localization, or affinity for DNA is directly or indirectly regulated by the level of oxidation (Liu et al. 2005; Lushchak 2010). However, it is still unclear how VDAC contributes to the redox states of the cytosol and mitochondria. It can be speculated that VDAC transports metabolites that participate in the determination of the redox states. For example, it is known that VDAC is involved in the superoxide anion release from mitochondria to cytosol (Han et al. 2003). Interestingly, the differences in the cytosol redox states observed for wild type and $\Delta$ porl cells in different growth phases coincide with the differences in levels of the superoxide anion release from their mitochondria (Budzinska et al. 2007, 2009). On the other hand, VDAC affects mitochondrial respiration activity as VDAC is necessary for metabolite passage across the mitochondrial outer membrane (Lee et al. 1998; Galganska et al. 2010). Accordingly, it has been observed that the respiratory activity is important for functions of Hap proteins, including Hap4 (Butow and Avadhani 2004). This in turn, could explain the effect of VDAC on levels of mRNA encoding Hap4. Similarly, VDAC clearly affects levels of mRNA encoding Rtg1 but the responsible mechanism does not involve changes of the intracellular redox states. Probably, intracellular metabolite levels known to be mediated by VDAC are relevant (e.g. Crespo et al. 2002), although partly the effect might result from changes of expression of Rtg2 that controls activity of Rtg1 (Butow and Avadhani 2004).

Summing up, the present work has revealed for the first time the important role of VDAC in controlling levels of mRNAs encoding nuclear proteins sensing the redox state (Sir2) and participating in adaptation to the redox state change (Skn7, Msn2, Msn4, Yap1, Yap2) as well as proteins involved in communication between mitochondria and the nucleus (Rtg1, Rtg2). The involved mechanism is generally based on changes of the intracellular redox states mediated by VDAC. However, VDAC appears also to be able to affect mRNA levels by mechanism not based on changes of the intracellular redox states, as shown for Rtg 1 and Hap4. Thus, in addition to regulating the metabolic and energetic functions of mitochondria, VDAC is involved in the communication between mitochondria and the rest of the cell, although future studies are required to fully describe this function of VDAC.

Acknowledgments The work was supported by grants from the Polish Ministry of Science and Higher Education (N N301 309837) and Foundation for Polish Science (POMOST_C/7).

Open Access This article is distributed under the terms of the Creative Commons Attribution Noncommercial License which permits any noncommercial use, distribution, and reproduction in any medium, provided the original author(s) and source are credited.

\section{References}

Aragon AD, Quiñones GA, Thomas EV, Roy S, Werner-Washburne M (2006) Genome Biol 7:R9

Benz R (1994) Biochim Biophys Acta 1197:167-196

Blachly-Dyson E, Forte M (2001) IUBMB Life 52:113-118

Blachly-Dyson E, Song J, Wolfgang WJ, Colombini M, Forte M (1997) Mol Cell Biol 17:5727-5738

Bohnert M, Pfanner N, van der Laan M (2007) FEBS Lett 581:2802-2810

Brons JF, De Jong M, Valens M, Grivell LA, Bolotin-Fukuhara M, Blom J (2002) Yeast 19:923-932 
Budzinska M, Galganska H, Wojtkowska M, Stobienia O, Kmita H (2007) Biochem Biophys Res Commun 357:1065-1070

Budzińska M, Gałgańska H, Karachitos A, Wojtkowska M, Kmita H (2009) J Bioenerg Biomembr 41:361-367

Butow RA, Avadhani NG (2004) Mol Cell 14:1-15

Cabiscol E, Piulats E, Echave P, Herrero E, Ros J (2000) J Biol Chem 275:27393-27398

Colombini M (2004) Mol Cell Biochem 256-257:107-115

Crespo JL, Powers T, Fowler B, Hall MN (2002) Proc Natl Acad Sci USA 99:6784-6789

Daum G, Gasser SM, Schatz G (1982) J Biol Chem 257:13075-13080

De Pinto V, Messina A, Accardi R, Aiello R, Guarino F, Tomasello MF, Tommasino M, Tasco G, Casadio R, Benz R, De Giorgi F, Ichas F, Baker M, Lawen A (2003) Ital J Biochem 52:17-24

Dolezal P, Likic V, Tachezy J, Lithgow T (2006) Science 313:314-318

Drakulic T, Temple MD, Guido R, Jarolim S, Breitenbach M, Attfield PV, Dawes IW (2005) FEMS Yeast Res 5:1215-2128

Galganska H, Budzinska M, Wojtkowska M, Kmita H (2008) Archiv Biochem Biophys 479:39-45

Galganska H, Karachitos A, Wojtkowska M, Stobienia O, Budzinska M, Kmita H (2010) Biochim Biophys Acta 1797:1276-1280

García-Rodríguez LJ, Gay AC, Pon LA (2007) J Cell Biol 176:197-207

Han D, Antunes F, Canali R, Rettori D, Cadenas E (2003) J Biol Chem 278:5557-5563

Huizing M, Ruitenbeek W, Thinnes FP, De Pinto V, Wendel U, Trijbels FJ, Smit LM, ter Laak HJ, van den Heuvel LP (1996) Pediatr Res 39:760-765
Kmita H, Antos N, Wojtkowska M, Hryniewiecka L (2004) J Bioenerg Biomembr 36:187-193

Lee AC, Xu X, Blachly-Dyson E, Forte M, Colombini M (1998) J Membr Biol 161:173-181

Lemasters JJ, Holmuhamedov E (2006) Biochim Biophys Acta 1762:181-190

Liu H, Colavitti R, Rovira II, Finkel T (2005) Circ Res 97:967-974

Lushchak V (2010) Biochemistry (Mosc) 75:281-296

Mannella CA, Kinnally KW (2008) J Bioenerg Biomembr 40:149-155

Mokranjac D, Neupert W (2009) Biochim Biophys Acta 1793:33-41

Pesaresi P, Schneider A, Kleine T, Leister D (2007) Curr Opin Plant Biol 10:600-606

Rodríguez-Quiñones JF, Irizarry RA, Díaz-Blanco NL, Rivera-Molina FE, Gómez-Garzón D, Rodríguez-Medina JR (2008) BMC Genomics 9:34

Rostovtseva TK, Bezrukov SM (2008) J Bioenerg Biomembr 40:163-170

Schein SJ, Colombini M, Finkelstein A (1976) J Membr Biol 30:99-120

Shoshan-Barmatz V, Keinan N, Zaid H (2008) J Bioenerg Biomembr 40:183-191

Shoshan-Barmatz V, De Pinto V, Zweckstetter M, Raviv Z, Keinan N, Arbel N (2010) Mol Aspects Med 31:227-285

Storz P (2006) Sci. STKE 332, re3

Sturtz LA, Diekert K, Jensen LT, Lill R, Culotta VC (2001) J Biol Chem 276:38084-38089

Walther DM, Rapaport D, Tommassen J (2009) Cell Mol Life Sci 66:2789-2804

Woodson JD, Chory J (2008) Nat Rev Genet 9:383-395 